\title{
Automatic Segmentation of Moving Objects By Fusing Multi-Information
}

\author{
Lin Zheng Chongzhao Han Dongguang Zuo Hongyan Zhu \\ School of Electronics \& information engineering, Xi' an Jiaotong University \\ Xi'an, Shaanxi, China \\ Linzheng@mailst.xjtu.edu.cn czhan@mail.xjtu.edu.cn zdg_1l_zly@263.net
}

\begin{abstract}
An automatic segmentation algorithm based on information fusion is presented. A frame difference method is used to get the initial contour of moving object. Simultaneously, region segmentation is applied on the current image according to the color information. By fusing the motion information, region information and the information of object's edge, an active contour method segments the moving object accurately. The experiments show that this segmentation algorithm performs robustly, and has a high accuracy.
\end{abstract}

Keywords: multi-information fusion; active contour; segmentation.

\section{Introduction}

Automatic segmentation of moving object is used for image coding, traffic surveillance and tracking, etc. In MPEG-4, the accuracy of segmentation decides the efficiency of video compression. And in surveillance, accurate detection of moving target makes the tracking more robust.

The accuracy of moving object segmentation is decided by the amount of information used. More video information is used, more accurate the segmentation will be. So in this paper, three kinds of video information are applied to this purpose, which are motion information, color information and edge information.

Firstly, motion information can be detected by using image difference method that is based on statistic model. Based on it, moving regions are separated from background. Secondly, color information can be obtained by establishing HIS color model, and used to segment the image into several regions. Then a color force is constructed according to the relative position between active contour model and those color regions. Thirdly, edge information is calculated by using image gradient method. And an edge-based image force can be got.

For fusing above information, an active contour model is proposed. Active contour model is a deformable curve, which would finally locate at the object' edge. There are two difficulties to use it. The first is the starting problem, and the second is how to catch the edge if the initial contour is far away from it.

In this paper, the initial active contour could be constructed by analyzing motion information, and use the edge of moving region as the initial contour.
The color information also creates a kind of external force, which is called color force. As the initial contour is far away from target's edge, the color force will attract it until it reach the nearby area.

The edge information contains accurate position information of edge. Then the image force is generated based on it. The image force has limited action range, so it couldn't play roles as the active contour is far away. On the other hand, if the active contour is firstly attracted to the nearby region by color force, the image force will play a main role to locate the contour on the accurate position.

This paper is arranged as following. In Section 2, motion information is analyzed. And the initial active contour model can be established. Then the color information is used to calculate color force, which will be discussed in Section 3. An information fusion system based on active contour model is constructed in Section 4. Finally, experiments show the good behave of this algorithm.

\section{Initial ACM Analysis based on motion information}

Initial ACM (active contour model) Analysis is an important step of moving target segmentation, and image difference method is used for this purpose. After the motion regions in the scene are found out, the edges of motion regions can be used as the initial ACM.

Normally, the regions of motion segmentation include the target in current frame and also previous frame. So the result of motion segmentation provides the coarse position and shape of moving target. Reference to image1, the motion segmentation consists of three parts:

$$
A=A_{1}+A_{2}+A_{3}
$$

where, $A_{1}$ is the newly uncovered background, $A_{2}$ is the still covered background. And $A_{3}$ is the newly covered background area.

If the newly uncovered background area $A_{1}$ is not such large, $A_{2}$ and $A_{3}$ will occupy a high ratio of occlusion area $A$. Since the moving target in current frame is constructed by $A_{2}$ and $A_{3}$, it is the favor to let $A$ as the coarse position of target. And its contour is used as the initial ACM. 
previous frame

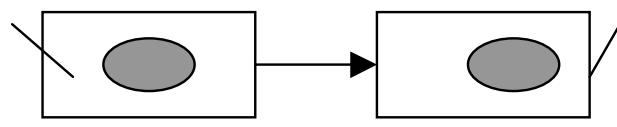

Difference Image

Figure1 Motion analysis by using Image Difference

The common methods for motion segmentation include image difference algorithm and optical flow. The optical flow method is over-computation. A normal image difference algorithm is sensitive to noise, and the threshold is difficult to determine. So a statistical-based image difference algorithm is used in this paper ${ }^{[1]}$.

Supposed that the camera is static during the target moves. The camera noise is uncorrelated between different frames, and let its variance is $\sigma_{1}^{2}$. Then a frame can be described as:

$$
I_{t}(k)=H_{t}(k)+n_{t}(k)
$$

where, $t$ denote the frame number, and $k$ is a pixel in the frame. $n_{t}(k)$ is the noise at pixel $k$ in frame $t, H_{t}(k)$ is the gray value if there is no noise.

The difference between current frame $t 2$ and previous frame $t 1$ is:

$$
D(k)=I_{t 2}(k)-I_{t 1}(k)=D_{H}(k)+D_{n}(k)
$$

where, $D_{H}(k)=H_{t 2}(k)-H_{t 1}(k)$ is a part of $D(k)$, which is caused by the uncovering or coverage during motion. $D_{n}(k)=n_{t 2}(k)-n_{t 1}(k)$

is cause by the camera noise.

According to the theory in [1], $D_{n}(k)$ obeys a zero mean Gaussian distribution $N(0, \sigma)$ with variance $\sigma^{2}$, which is twice the variance of the assumed Gaussian camera noise distribution :

$$
\sigma^{2}=2 \sigma_{1}^{2}
$$

if there is no movement at pixel $k, D_{n}(k)$ becomes

$$
p\left(D(k) \mid H_{0}\right)=\frac{1}{\sqrt{2 \pi \sigma^{2}}} \exp \left\{-\frac{D(k)^{2}}{2 \sigma^{2}}\right\}
$$

where, $H_{0}$ means that there are not movement at pixel $k$, same as $D_{H}(k)=0$. So $p\left(D(k) \mid H_{0}\right)$ is decided by the square of normalized image difference $(D(k) / \sigma)^{2}$. If there is somewhat movement at pixel $k$, normally $D_{H}(k)>>D_{n}(k)$, and then

$$
(D(k) / \sigma)^{2}>\left(D_{n}(k) / \sigma\right)^{2}
$$

So $(D(k) / \sigma)^{2}$ can be used for distinguishing moving pixels and static pixels.

If a pixel is belong to the moving region, the pixels close to it may be belong this region too, vice versa. So a set of image difference inside a small decision region instead of only a single pixel is evaluated, which makes the movement detection more reliable. We thus compute the local sum $\Delta^{2}(k)$ of $(D(k) / \sigma)^{2}$ inside the small sliding window $\varpi_{k}$, with $k$ denoting the center pixel of window $\varpi_{k}$ :

$$
\Delta^{2}(k)=\sum_{i \in \bar{\pi}_{k}}\left(\frac{D(i)}{\sigma}\right)^{2}
$$

If there is no movement in $\varpi_{k}$, every normalized difference $D(i) / \sigma, i \in \varpi_{k}$ obeys the Gaussian distribution $N(0,1)$. Thus $\Delta^{2}(k)$ obeys $\chi_{m}^{2}$-distribution with as many degrees of freedom as there are pixels inside the window. With the distribution $p\left(\Delta^{2} \mid H_{0}\right)$ known, the movement area in current frame can be detected out.

Then a suitable little value is selected for the significance level $\alpha$. And a corresponding threshold $l_{\alpha}$ is computed according to

$$
\alpha=p\left(\Delta^{2}>l_{\alpha} \mid H_{0}\right)
$$

The statistic can be evaluated at every pixel $k$ of the current frame, and whenever it exceeds $l_{\alpha}$, the corresponding pixel is marked as movement, otherwise as static.

After the moving region is obtained, an edge detection algorithm is used to get the initial ACM. Above algorithm can be conclude as following

(1) Selects a suitable $\alpha$, which may be varied between $10^{-6}$ and $10^{-2}$. Then the corresponding $l_{\alpha}$ is computed

(2) Computes the difference image $D(k)$ between current frame and previous frame

(3) Calculates the variance $\sigma^{2}$ of camera noise. Since there may be no movement at some regions, such as those near corners or edges, $\sigma^{2}$ could be evaluated in one of these region on difference image $D(k)$

(4) Computes $\Delta^{2}(k), k \in D$. If $\Delta^{2}(k)>l_{\alpha}$, the pixel belong to moving area, otherwise belong to static background.

(5) Processes the moving area with morphologic method.

(6) Detects the edges of movement areas, which are used as the initial ACM. 


\section{Color force analysis based on color information}

During the process of automatic segmentation, Color information could perform important roles. This is based on the following two aspects. Firstly, moving targets normally have different color with background. Secondly, color images contain more information than in gray images. So the color information is used.

A lot of Color image segmentation methods have been provided, which include clustering algorithm, region split-merge method, Markov Random field method, etc ${ }^{[2]}$. These methods may be accurate but somewhat complex. Since it is unnecessary to have an accurate color segment result in this fusion method, a coarse but rapid method is used, which is base on HIS color model ${ }^{[3]}$.

In HIS model, I denotes intensity, $\mathrm{H}$ denotes Hue, and $\mathrm{S}$ denotes saturation. The main advantage of HIS model than RGB model is that it obeys visual character of human.

Among the three components of HIS color model, the $\mathrm{I}$ and $\mathrm{H}$ components can have some corresponding interpretations in their own space. While different I values represent different levels of shades of gray perceived by human beings, the different $H$ values represent the different dominant color perceived by human beings. The third component, S, refers to the relative color purity. In other words, the degree of saturation is inversely proportional to the amount of white light mixed with a hue. On one extreme, the pure spectrum colors are fully saturated. In such a case, different colors are totally discriminated by the $\mathrm{H}$ component of a color image. On the other extreme, the saturation of a gray image is zero. In such a case, only the I component of a color image has some discrimination power.

According to this idea, color image can be firstly segmented by the $\mathrm{S}$ component. Then the segment results could be finely segmented by $\mathrm{H}$ or $\mathrm{S}$ component respectively. The algorithm is following:

(1) Analyzes the histogram of $\mathrm{S}$ component. Then a threshold is found out, which is closest to the average gray level of $\mathrm{S}$. As a result, the $\mathrm{S}$ image is divided into two images, one contains high saturation regions $S_{h}$, and another contains low saturation regions $S_{l}$.

(2) using $\mathrm{H}$ component finely segments the high saturation image $S_{h}$, a histogram method is also used here.

(3) Using the I component finely segments the low saturation i mage $S_{l}$.

(4) Since $S_{h}$ and $S_{l}$ have not any overlapped parts, the segmentation results of step 2 and step 3 could be merged diretely, which is just the segment of original color image.

In the process of threshold detection, the histogram maybe has a single peak, with a local flattening or 'shoulder' on one side. Two reasons contribute to this. Firstly, the object and background have nearly identical gray-level distributions. Secondly, the object is so small that submerged by background. To solve this problem, an improved upper convex hull method is present here, which is based on concavity measure of histogram.

(1) the histogram $h(z)$ is treated as a region in the plane, and then the upper convex hull $h^{\prime}(z)$ can be $\operatorname{got}^{[4]}$.

(2)Calculates the convex hull residual :

$$
r(z)=h^{\prime}(z)-h(z)
$$

(3) Removes the local maximum value of $r(z)$, with a $1-D$ median filter. Then gets the improved convex hull residual image $r^{\prime}(z)$.

(4) Calculate the maxima of $r^{\prime}(z)$, which are selected as threshold of input image. Since median filter could cause some continue maxima, the middle point of them is selected as valid.

Followed above methods, every color region of targets or background has been detected. Referring to figure 1, all color regions of target must appear in the moving region. But some reasons may cause mistakes. In the process of motion segmentation, the result is decided by noise statistic character. As the character varies, the segmentation may become inaccurate. On the other hand, the image noise can affect the accuracy of color segmentation. Considering above reasons, some parts of target appear outside of the motion segmentation. With the same reason, some parts of background may become inside of motion segmentation. For an accurate segmentation, these mistakes must be corrected. On the other hand, in figure 1, some background regions are also appeared in the segmentation because of newly uncovering, these regions are also need to be cleaned.

So, in this paper, an active contour model is applied to remove all background regions from motion segmentation, and let all those target regions into the segmentation. Based on this idea, a color force is proposed.

Supposed that active contour model is intersecting with a region $\mathrm{A}$, which has an area $A_{\text {inner }}$ inside the active contour. The ratio of occupance is :

$$
\text { Ratio }=\frac{A_{\text {inner }}}{A}
$$

Defines $\vec{N}$ as the normal at a control point of active contour model, which directs outward. Then all control points inside region $A$ has such kind of color force :

$$
F_{\text {color }}=-\sin (2 \pi \text { Ratio }) \vec{N}
$$

just that, they have the equal color force, but direct differently along their normal respectively.

Referring to figure 2, when active contour model is located inside a target region, normally Ratio $>0.5$, and color force directs outward, then force the active contour expanding. When active contour model is located in a 
backgroud region, normally have Ratio $<0.5$, so color force directs inward, and let the active contour contracting. When Ratio $=0.5$, it is difficult to decide, so color force is zero.
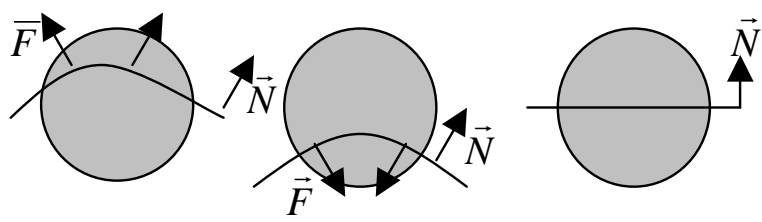

(a) Ratio $>0.5$ (b) Ratio $<0.5$

(c) Ratio $=0.5$

Figure2. the color force on different condition

This color force has some characters. As the active contour is far away from the region's edge, it is strong, and plays a main role in forcing active contour model moving to the edge. As the active contour close to the region's edge, it becomes weak. This phenomenon is consistent with the inaccuracy of color segmentation. But at this time, edge information become stronger, and plays an important role to locate the active contour.

So we can conclude that, the color force and edgebased image force compensate each other on action distance and location accuracy.

\section{ACM algorithm by fusing multi- information}

In this paper, active contour model is used to fuse three kinds of information, which are motion information, color information and edge-based image force. Then the moving target is segmented based on above information.

\subsection{Theory of active contour}

Active contour is an energy-minimizing parametric curve that deforms to fit the image characters. It can be expressed as: $v(s)=(x(s), y(s)), s \in[0,1]$, which is either open or close ${ }^{[5]}$. Energy of an active contour contains two part :

$$
E_{\text {snake }}=E_{\text {int }}+E_{\text {ext }}
$$

where, $E_{\text {int }}$ is the inner energy, which make active contour smooth. $E_{\text {int }}$ also consists of two parts :

$$
E_{\text {int }}=\int_{0}^{1} \alpha\left|\frac{\partial v}{\partial s}\right|^{2}+\beta\left|\frac{\partial^{2} v}{\partial s^{2}}\right|^{2} d s
$$

, which controls tension and stiff respectively.

$E_{e x t}$ is external energy that pushes active contour to image characters. Since edge is an important image character, the Edge-based energy $E_{\text {image }}$ becomes the main part of $E_{e x t}$, and have the following expression

$$
E_{\text {image }}=-\int_{0}^{1}\|\nabla I(v)\|^{2} d s
$$

As $E_{\text {snake }}$ reach the minimum value, active contour arrives at the target. And it satisfies the following EulerLagrange equation:

$$
0=\frac{\partial v}{\partial t}=\alpha \frac{\partial^{2} v}{\partial s^{2}}-\beta \frac{\partial^{4} v}{\partial s^{4}}+F_{e x t}
$$

where, $F_{\text {ext }}=F_{\text {image }}=-\nabla E_{\text {image }}$ is a differentiable external force. We discretizes the equation (9) by finite differences, then an iterative equation is got :

$$
V^{k+1}=(I+\tau A)^{-1}\left(V^{k}+F_{e x t}\left(V^{k}\right)\right)
$$

where, $V^{k+1}$ and $V^{k}$ is $M \times 2$ position vector in $(k+1)$ th and $k$ th iteration respectively. $M$ is the number of control points. $\tau$ is the step. $A$ is a $M \times M$ metrix that is formed by $\alpha, \beta$ and $\tau$.

\subsection{The theory of segmentation based on multi-information}

If an active contour is used for movement segmentation, there are two problems need to be solved. The first is how to get the initial active contour. Although interactive method could be used, it is not automatically. The second problem is that if the initial active contour is far away from the edges, the active contour may be catch by a false local minimum. The reason for this is action distance limitation of image energy.

For conquering above problems, movement information is used to supply the initial active contour. And color information is applied to catch a distant active contour. As a result, the active contour deforms to the nearby region of edges. Then attracted by the edge information, active contour arrives at characters accurately.

The flowchart of above algorithm is:

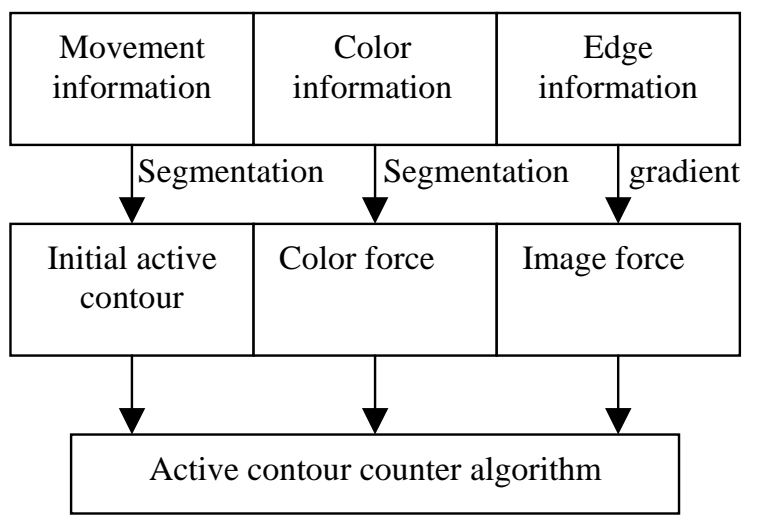

Figure 3 The ACM algorithm based on information fusion

In the flowchart, initial active contour and edge-based image energy could be calculated once time. But color force need to be evaluated every iteration. Since the color segmentation is coarse, the color segmentation maybe contains not such more regions. And so on, the regions that intersect with active contour are also less. So the computation for color force is not heavy. 
Like the balloon force in [6], color force can be add into $F_{\text {ext }}$ directly:

$$
F_{\text {ext }}=\rho F_{\text {color }} /\left|F_{\text {color }}\right|+\gamma F_{\text {image }} /\left|F_{\text {image }}\right|
$$

in practice, $\gamma=0.7 \sim 1$, and $\rho=1$.

\section{Experiments and result analysis}

There are two experiments in this paper. In the first experiment, Mom head-shoulder sequence is used. Among the sequence, Mom's head turns around very slowly. Referring to figure4.

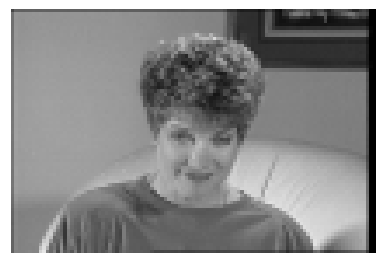

(a)2th frame in Mom sequence

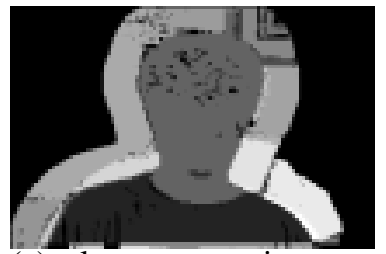

(c)color segmentation

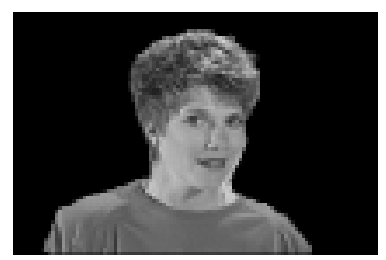

(e) segmentation of 95th frame (f) segmentation of 114th frame

Figure4 segment of the woman in Mom sequence

This experiment shows that the automatic segmentation based on information fusion could accuratly segment the woman.

In experiment 2 , traffic sequence is used. In frame 425, a white car moves upward along the right road. Automatic segmentation algorithm of this paper is used to tracking this car for about 60 frames. The segmentation and tracking result Refers to figure5.

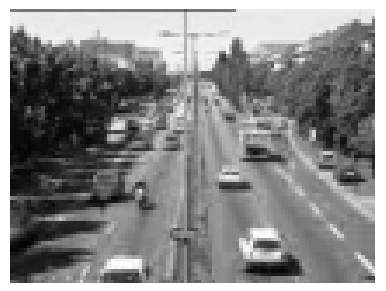

(a)the 425th frame

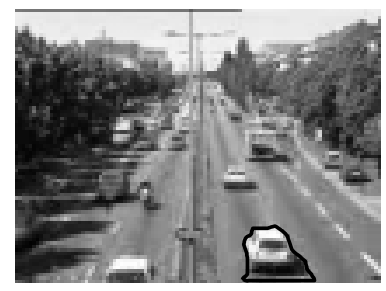

(b)initial active contour in current frame

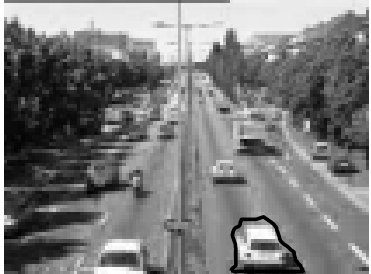

(c)initial active contour in previous frame

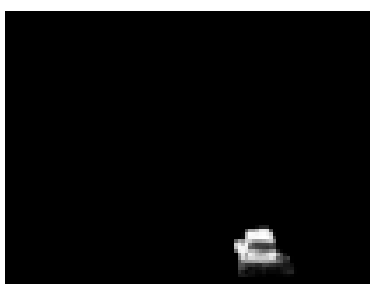

(e) segment result using our method

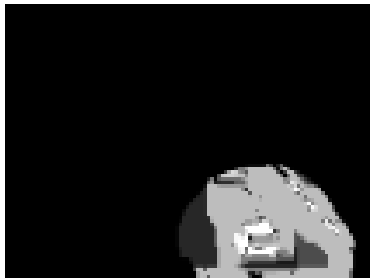

(d)color segmentation

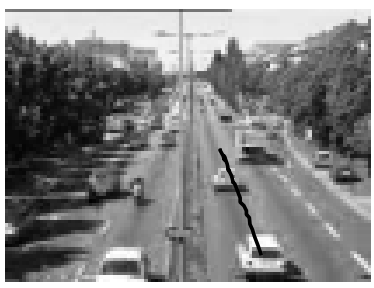

(f)the tracking trajectory(black) from frame 425 to 484
Figure5 segment and tracking car in Traffic sequence

In Figure5(b), the black contour that surrounds white car is the initial active contour. It is obviously that white car lies at the top of initial contour, and a gap at the bottom of active contour. In Figure 5(c), the initial active contour is placed in previous frame. At that case, white car lies at the bottom of contour, and a gap at the top of active contour. In Figure 5(e), the car is segmented out by using our method. In this case, the contour fits the car very well.

Figure5 (b), (c), (e) show that the motion segmentation not only contains the target in current frame, but also the target in previous frame. During the deforming of active contour model, the regions belong to previous frame but current frame has been removed.

Referring to figure 5(6), the tracking is stable.

\section{Conclusion}

In this paper, an automatic segmentation algorithm of moving objects based on information fusion is proposed. The useful information include movement, color, edge, which is fused by active contour model.

The experiments show that the algorithm has a good behave on target segmentation, and tracks target reliably.

\section{Reference}

[1] T. Aach, A. Kaup. Statistical model-based change detection in moving video. Signal Processing. 1993,31:165-180.

[2] Y.J.Zhang. Image Segmentation. China Science Press. $2001,116 \cdot 117$

[3] Y.J.Zhang, Y.R.Yao. Automatic Face Segmentation Using Color Cues for Coding for Coding Typical Videophone Scenes. SPIE,1997,3024:468-479.

[4] A.Rosenfeld, P.D.Torre. Histogram Concavity Analysis as an Aid in Threshold Selection. IEEE Trans 
on Systems, Man, And Cybernetics.1983, vol.SMC-13.

No.3:231-629

[5] M.Kass, A.Witkin, D.Terzopoulos. Snakes:Active Contour Models. Int J. Computer Vision. 1987:321331

[6] L.D.Cohen. On Active Contour Models and Ballons. CVGIP(53), 1991 March, No. 2,: 211-218 